\title{
ON MULTIPARAMETER DISTRIBUTIONS OF ORDER $k$
}

\author{
ANDREAS N. PHILIPPOU \\ Department of Mathematics, University of Patras, Patras, Greece
}

(Received April 16, 1987; revised August 14, 1987)

\begin{abstract}
A multiparameter negative binomial distribution of order $k$ is obtained by compounding the extended (or multiparameter) Poisson distribution of order $k$ by the gamma distribution. A multiparameter logarithmic series distribution of order $k$ is derived next, as the zero truncated limit of the first distribution. Finally a few genesis schemes and interrelationships are established for these three multiparameter distributions of order $k$. The present work extends several properties of distributions of order $k$.
\end{abstract}

Key words and phrases: Multiparameter distributions of order $k$, type I and type II distributions of order $k$, genesis schemes and interrelationships, extended distributions of order $k$.

\section{Introduction}

The exact distribution theory of the discrete distributions of order $k$ was initiated by Philippou et al. (1983), who introduced the geometric distribution of order $k$ (see also Philippou and Muwafi (1982)), and derived from its study the negative binomial and Poisson distributions of the same order. Further results and / or new distributions of order $k$ were obtained by Philippou (1983, 1984), Aki et al. (1984), Aki (1985), Charalambides (1986), Hirano (1986), Philippou and Makri (1986) and Xekalaki et al. (1987). Motivated by Philippou (1983), who obtained a new negative binomial (or compound Poisson) distribution of order $k$, we presently derive a multiparameter negative binomial distribution of order $k$, by compounding the extended (or multiparameter) Poisson distribution of order $k$ of Aki (1985). We also derive a multiparameter logarithmic series distribution of order $k$, as the zero truncated limit of the first, and we examine some genesis schemes and interrelationships of the three distributions.

All the propositions of the present paper may be specialized in an obvious manner to respective distributions of order $k$ to give known (or even some new) results. We shall not do this here, however, for space economy. Also, in order to avoid unnecessary repetitions, we mention here that in this paper 
$x_{1}, \ldots, x_{k}$ are non-negative integers as specified.

2. Multiparameter negative binomial and logarithmic series distributions of order $k$

We first consider the following;

DEFINITION 2.1. A random variable (rv) $X$ is said to have the Poisson distribution of order $k$ with parameters $\lambda_{1}, \ldots, \lambda_{k}\left(\lambda_{i}>0\right.$ for $\left.1 \leq i \leq k\right)$, to be denoted by $P_{k}\left(\lambda_{1}, \ldots, \lambda_{k}\right)$, if

$$
P(X=x)=\sum_{\substack{x_{1} \\ x_{1}+2 x_{2}+\cdots, \cdots+x_{k} \exists \\ x_{k}=x}} \frac{\lambda_{1}^{x_{1}} \cdots \lambda_{k}^{x_{k}}}{x_{1} ! \cdots x_{k} !} \exp \left(-\sum_{i=1}^{k} \lambda_{i}\right), \quad x=0,1, \ldots .
$$

This distribution, which is due to Adelson (1966), was called "stuttering" Poisson by Johnson and Kotz (1969) and "extended Poisson distribution of order $k$ " by Aki (1985). We shall use it in Proposition 3.1 of Philippou (1983), in place of the Poisson distribution of order $k$ with parameter $\lambda$, to derive a multiparameter negative binomial distribution of order $k$.

Proposition 2.1. Let $X$ and $Y$ be two rvs such that $X \mid Y=y$ is distributed as $P_{k}\left(\lambda_{1} y, \ldots, \lambda_{k} y\right)$ and $f_{Y}(y)=\alpha^{r} y^{r-1} e^{-\alpha y} / \Gamma(r)(y, \alpha$ and $r$ positive), and set $q_{i}=\lambda_{i} /\left(\lambda_{1}+\cdots+\lambda_{k}+\alpha\right)(1 \leq i \leq k)$ and $p=1-q_{1}-\cdots-q_{k}$. Then

$$
\begin{aligned}
& P(X=x)=p^{r} \sum_{\substack{x_{1}+2 x_{2}, \cdots, \cdots, x_{3} 3 \\
x_{k}=x}} \frac{\Gamma\left(x_{1}+\cdots+x_{k}+r\right)}{x_{1} ! \cdots x_{k} ! \Gamma(r)} q_{1}^{x_{1}} \cdots q_{k}^{x_{k}}, \\
& x=0,1, \ldots .
\end{aligned}
$$

Proof. We have

$$
\begin{aligned}
& P(X=x)=\int_{0}^{\infty} \sum_{\substack{x_{1}, \ldots, x_{3}, 3 \\
x_{1}+2 x_{2}+\cdots+k x_{k}=x}} \frac{\left(\lambda_{1} y\right)^{x_{1}} \cdots\left(\lambda_{k} y\right)^{x_{k}}}{x_{1} ! \cdots x_{k} !} \\
& \times\left[\exp \left(-y \sum_{i=1}^{k} \lambda_{i}\right)\right] \frac{\alpha^{r} y^{r-1}}{\Gamma(r)} e^{-\alpha y} d y \\
& =\alpha^{r} \sum_{\substack{\left.x_{1}, \ldots, x_{k}\right) \\
x_{1}+2 x_{2}+\cdots+x_{k}=x}} \frac{\lambda_{1}^{x_{1}} \cdots \lambda_{k}^{x_{k}}}{x_{1} ! \cdots x_{k} ! \Gamma(r)} \\
& \times \int_{0}^{\infty} y^{x_{1}+\cdots+x_{k}+r-1} e^{-\left(\lambda_{1}+\cdots+\lambda_{k}+\alpha\right) y} d y,
\end{aligned}
$$


and

$$
\int_{0}^{\infty} y^{x_{1}+\cdots+x_{k}+r-1} e^{-\left(\lambda_{1}+\cdots+\lambda_{k}+\alpha\right) y} d y=\frac{\Gamma\left(x_{1}+\cdots+x_{k}+r\right)}{\left(\lambda_{1}+\cdots+\lambda_{k}+\alpha\right)^{x_{1}+\cdots+x_{k}+r}},
$$

from which the proposition follows.

We introduce now the following;

DEFINITION 2.2. A rv $X$ is said to have the negative binomial distribution of order $k$ with parameters $r, q_{1}, \ldots, q_{k}\left(r>0,0<q_{i}<1\right.$ for $1 \leq i \leq k$ and $\left.q_{1}+\cdots+q_{k}<1\right)$, to be denoted by $\mathrm{NB}_{k}\left(r ; q_{1}, \ldots, q_{k}\right)$, if

$$
\begin{aligned}
P(X=x)=p^{r} \sum_{\substack{x_{1} \\
x_{1}+2 x_{2}+\ldots, \ldots+k x_{k}, x_{k}=x}} \frac{\Gamma\left(x_{1}+\cdots+x_{k}+r\right)}{x_{1} ! \cdots x_{k} ! \Gamma(r)} q_{1}^{x_{1}} \cdots q_{k}^{x_{k}}, & \\
x & =0,1, \ldots,
\end{aligned}
$$

where $p=1-q_{1}-\cdots-q_{k}$.

If $q_{i}=P^{i-1} Q(1 \leq i \leq k)$ so that $p=P^{k}$, we observe that $\mathrm{NB}_{k}\left(r ; q_{1}, \ldots, q_{k}\right)=$ $\overline{\mathrm{NB}}_{k, \mathrm{I}}(r, P)$, where $\overline{\mathrm{NB}}_{k, \mathrm{I}}(r, P)$ is the shifted negative binomial distribution of order $k$ (Philippou et al. (1983) and Aki et al. (1984)). Here and in the sequel $Q=1-P$. Furthermore, if $q_{i}=q / k(1 \leq i \leq k)$, we note that $\mathrm{NB}_{k}\left(r ; q_{1}, \ldots, q_{k}\right)=$ $\mathrm{NB}_{k, \mathrm{II}}(r, p)$, where $\mathrm{NB}_{k, \mathrm{II}}(r, p)$ denotes the compound Poisson (or negative binomial) distribution of order $k$ (Philippou (1983)), with $p=\alpha / \alpha+k$. As it was mentioned in Philippou (1983), this distribution is different than the corresponding one of Philippou et al. (1983). We call it negative binomial distribution of order $k$, type II, with parameters $r$ and $p$.

We proceed now to obtain a multiparameter logarithmic series distribution of order $k$.

PROPOSITION 2.2. Let $X$ be a $r v$ distributed as $\mathrm{NB}_{k}\left(r ; q_{1}, \ldots, q_{k}\right)$, and assume that $r \rightarrow 0$. Then, for $x=1,2, \ldots$,

$$
P(X=x \mid X \geq 1) \rightarrow \alpha \underset{\substack{x_{1} \\ x_{1}+2 x_{2}+\cdots, x_{k} 3 \\ x_{k}=x}}{ } \frac{\left(x_{1}+\cdots+x_{k}-1\right) !}{x_{1} ! \cdots x_{k} !} q_{1}^{x_{1}} \cdots q_{k}^{x_{k}}
$$

where $\alpha=-(\log p)^{-1}$.

ProOF. For $x=1,2, \ldots$, we have 


$$
\begin{aligned}
P(X & =x \mid X \geq 1) \\
& =\frac{P(X=x, X \geq 1)}{1-P(X=0)} \\
& =\frac{p^{r}}{1-p^{r}} \sum_{\substack{x_{1}+2 x_{2}+\ldots, \ldots+k x_{k}=x \\
x_{1}, \ldots, x_{k},}} \frac{\Gamma\left(x_{1}+\cdots+x_{k}+r\right)}{x_{1} ! \cdots x_{k} ! \Gamma(r)} q_{1}^{x_{1}} \cdots q_{k}^{x_{k}},
\end{aligned}
$$

$$
\rightarrow-(\log p)^{-1} \sum_{\substack{x_{1}, \ldots, x_{k} \rightrightarrows \\ x_{1}+2 x_{2}+\ldots+k x_{k}=x}} \frac{\left(x_{1}+\cdots+x_{k}-1\right) !}{x_{1} ! \cdots x_{k} !} q_{1}^{x_{1}} \cdots q_{k}^{x_{k}}
$$

which establishes the proposition.

DEFINITION 2.3. A rv $X$ is said to have the logarithmic series distribution of order $k$ with parameters $q_{1}, \ldots, q_{k}\left(0<q_{i}<1\right.$ for $1 \leq i \leq k$ and $\left.q_{1}+\cdots+q_{k}<1\right)$, to be denoted by $\operatorname{LS}_{k}\left(q_{1}, \ldots, q_{k}\right)$, if

$$
\begin{aligned}
& P(X=x)=\alpha \underset{\substack{x_{1} \ldots, x_{k} \geqslant \\
x_{1}+2 x_{2}+\cdots+k x_{k}-x}}{\sum} \frac{\left(x_{1}+\cdots+x_{k}-1\right) !}{x_{1} ! \cdots x_{k} !} q_{1}^{x_{1}} \cdots q_{k}^{x_{k}} \\
& x=1,2, \ldots,
\end{aligned}
$$

where $\alpha=-(\log p)^{-1}$ and $p=1-q_{1}-\cdots-q_{k}$.

If $q_{i}=P^{i-1} Q(1 \leq i \leq k)$ so that $p=P^{k}$, we observe that $\operatorname{LS}_{k}\left(q_{1}, \ldots, q_{k}\right)=$ $\mathrm{LS}_{k, \mathrm{I}}(P)$, where $\mathrm{LS}_{k, \mathrm{I}}(P)$ is the logarithmic series distribution of order $k$ of $\mathrm{Aki}$ et al. (1984). Furthermore, if $q_{i}=q / k(1 \leq i \leq k)$, we note that $\operatorname{LS}_{k}\left(q_{1}, \ldots, q_{k}\right)$ reduces to a logarithmic series distribution of order $k$, different than the one of Aki et al. (1984). We call it logarithmic series distribution of order $k$, type II, with parameter $q$, and denote it by $\operatorname{LS}_{k, \mathrm{II}}(q)$.

Remark 2.1. By setting $q_{1}=Q_{1}$ and $q_{i}=P_{1} \cdots P_{i-1} Q_{i}(2 \leq i \leq k)$, which imply $p=P_{1} \cdots P_{k}$, we observe that

$$
\mathrm{NB}_{k}\left(r ; q_{1}, \ldots, q_{k}\right)=\overline{\mathrm{ENB}}_{k}\left(r ; P_{1}, \ldots, P_{k}\right),
$$

and

$$
\mathrm{LS}_{k}\left(q_{1}, \ldots, q_{k}\right)=\mathrm{ELS}_{k}\left(P_{1}, \ldots, P_{k}\right)
$$

where $\overline{\operatorname{ENB}}_{k}\left(r ; P_{1}, \ldots, P_{k}\right)\left(\operatorname{ELS}_{k}\left(P_{1}, \ldots, P_{k}\right)\right)$ is the (suitably shifted) extended negative binomial (logarithmic series) distribution of order $k$ of Aki (1985). Here $Q_{i}=1-P_{i}(1 \leq i \leq k)$. 
The following representations are direct consequences of Definitions 2.1-2.3.

Proposition 2.3. Let $X_{i}(1 \leq i \leq k)$ be rvs and set $X=\sum_{i=1}^{k} i X_{i}$. Then

(a) $X$ is distributed as $P_{k}\left(\lambda_{1}, \ldots, \lambda_{k}\right)$ if and only if $X_{i}(1 \leq i \leq k)$ are distributed independently as $P_{1}\left(\lambda_{i}\right)$;

(b) $X$ is distributed as $\mathrm{NB}_{k}\left(r ; q_{1}, \ldots, q_{k}\right)$ if and only if $X_{1}, \ldots, X_{k}$ are jointly distributed as multivariate negative binomial with parameters $r$, $q_{1}, \ldots, q_{k}$

(c) $X$ is distributed as $\operatorname{LS}_{k}\left(q_{1}, \ldots, q_{k}\right)$ if and only if $X_{1}, \ldots, X_{k}$ are jointly distributed as multivariate logarithmic series with parameters $q_{1}, \ldots, q_{k}$.

Upon using Proposition 2.3(a), we get;

PROPOSITION 2.4. Let $X$ be a rv distributed as $P_{k}\left(\lambda_{1}, \ldots, \lambda_{k}\right)$. Then

(a) $g_{X}(t)=\exp \sum_{i=1}^{k} \lambda_{i}\left(t^{i}-1\right), \quad|t| \leq 1$;

(b) $E(X)=\sum_{i=1}^{k} i \lambda_{i}$ and $\sigma^{2}(X)=\sum_{i=1}^{k} i^{2} \lambda_{i}$.

Upon using simple expectation properties and Propositions 2.1 and 2.4, we get;

Proposition 2.5. Let $X$ be a rv distributed as $\mathrm{NB}_{k}\left(r ; q_{1}, \ldots, q_{k}\right)$. Then

(a) $g_{X}(t)=p^{r}\left(1-\sum_{i=1}^{k} q_{i t} t^{-r}, \quad|t| \leq 1\right.$;

(b) $E(X)=\frac{r}{p} \sum_{i=1}^{k} i q_{i} \quad$ and $\quad \sigma^{2}(X)=\frac{r}{p}\left[\sum_{i=1}^{k} i^{2} q_{i}+\frac{1}{p}\left(\sum_{i=1}^{k} i q_{i}\right)^{2}\right]$.

Upon using Definition 2.2, the transformation $x_{i}=n_{i}(1 \leq i \leq k)$ and $x=n+\sum_{i=1}^{k}(i-1) n_{i}$, and the multinomial theorem, we get part (a) of the following proposition.

Proposition 2.6. Let $X$ be a rv distributed as $\mathrm{LS}_{k}\left(q_{1}, \ldots, q_{k}\right)$. Then

(a) $g_{X}(t)=-\alpha \log \left(1-\sum_{i=1}^{k} q_{i} t^{i}\right), \quad|t| \leq 1$;

(b) $E(X)=\frac{\alpha}{p} \sum_{i=1}^{k} i q_{i}$ and $\sigma^{2}(X)=\frac{\alpha}{p}\left[\sum_{i=1}^{k} i^{2} q_{i}+\frac{1-\alpha}{p}\left(\sum_{i=1}^{k} i q_{i}\right)^{2}\right]$.

We get part (b) by straightforward differentiation of $g_{X}(t)$.

Proposition 2.5(a) implies the following 
Proposition 2.7. Let $X_{i}(1 \leq i \leq n)$ be independent rvs distributed as $\mathrm{NB}_{k}\left(r_{i} ; q_{1}, \ldots, q_{k}\right)$, and set $X=X_{1}+\cdots+X_{n}$ and $r=r_{1}+\cdots+r_{n}$. Then $X$ is distributed as $\mathrm{NB}_{k}\left(r ; q_{1}, \ldots, q_{k}\right)$.

\section{Genesis schemes and interrelationships}

In this section we present a few genesis schemes and interrelationships for the three multiparameter distributions of order $k$. We start with an urn model which gives rise to $\mathrm{NB}_{k}\left(r ; q_{1}, \ldots, q_{k}\right)$ with positive integer $r$.

PROPOSITION 3.1. An urn contains balls bearing the letters $F_{1}, \ldots, F_{k}$ and $S\left(\equiv S_{0}\right)$ with respective proportions $q_{1}, \ldots, q_{k}$ and $p\left(0<q_{i}<1\right.$ for $1 \leq i \leq k$, $q_{1}+\cdots+q_{k}<1$ and $\left.q_{1}+\cdots+q_{k}+p=1\right)$. Balls are drawn from the urn with replacement until $r$ balls $(r \geq 1)$ bearing the letter $S$ appear. Let $X$ be a $r v$ denoting the sum of the indices of the letters on the balls drawn. Then $X$ is distributed as $\mathrm{NB}_{k}\left(r ; q_{1}, \ldots, q_{k}\right)$.

PROOF. For any fixed non-negative integer $x$, a typical element of the event $(X=x)$ is an arrangement $a_{1} a_{2} \cdots a_{x_{1}+\cdots+x_{k}+r-1} S$ of the letters $F_{1}, \ldots, F_{k}$ and $S$, such that $r-1$ of the $a$ 's are $S, x_{i}$ of the $a$ 's are $F_{i}(1 \leq i \leq k)$, and $x_{1}+2 x_{2}+\cdots+k x_{k}=x$. Fix $x_{1}, \ldots, x_{k}(r$ is fixed). Then the number of the above arrangements is

$$
\left(\begin{array}{c}
x_{1}+\cdots+x_{k}+r-1 \\
x_{1}, \ldots, x_{k}, r-1
\end{array}\right),
$$

and each one of them has probability

$$
p^{r} q_{1}^{x_{1}} \cdots q_{k}^{x_{k}}
$$

But the non-negative integers $x_{1}, \ldots, x_{k}$ may vary subject to the condition $x_{1}+2 x_{2}+\cdots+k x_{k}=x$. Therefore

$$
\begin{aligned}
& P(X=x)=p^{r} \sum_{\substack{x_{1} \\
x_{1}+2 x_{2}, \cdots, \cdots, x_{k}, x_{k}=x}}\left(\begin{array}{c}
x_{1}+\cdots+x_{k}+r-1 \\
x_{1}, \ldots, x_{k}, r-1
\end{array}\right) q_{1}^{x_{1}} \cdots q_{k}^{x_{k}} \\
& x=0,1, \ldots .
\end{aligned}
$$

which establishes the proposition.

The next proposition provides a genesis scheme for $P_{k}\left(\lambda_{1}, \ldots, \lambda_{k}\right)$, related to $\mathrm{NB}_{k}\left(r ; q_{1}, \ldots, q_{k}\right)$.

Proposition 3.2. Let $X_{r}$ and $X$ be rvs distributed as $\mathbf{N B}_{k}\left(r ; q_{1}, \ldots, q_{k}\right)$ and $P_{k}\left(\lambda_{1}, \ldots, \lambda_{k}\right)$, respectively. Assume that $q_{i} \rightarrow 0$ and $r q_{i} \rightarrow \lambda_{i}(1 \leq i \leq k)$ as $r \rightarrow \infty$. 
Then

$$
P\left(X_{r}=x\right) \rightarrow P(X=x), \quad x=0,1, \ldots .
$$

Proof. For $|t| \leq 1$, we have

$$
\begin{aligned}
g_{X}(t) & =p^{r}\left(1-\sum_{i=1}^{k} q_{i} t^{i}\right)^{-r} \quad \text { (by Proposition 2.5(a)) } \\
& =\left(1-\frac{\sum_{i=1}^{k} r q_{i}}{r}\right)^{r}\left(1-\frac{\sum_{i=1}^{k} r q_{i} t^{i}}{r}\right)^{-r}, \text { since } p=1-\sum_{i=1}^{k} q_{i}, \\
& \rightarrow \exp \sum_{i=1}^{k} \lambda_{i}\left(t^{i}-1\right),
\end{aligned}
$$

which establishes the proposition, by means of Proposition 2.4(a).

It is well known that the negative binomial distribution results if the Poisson distribution with mean $-r \log p$ is generalized by the logarithmic series distribution. We now show that this scheme carries over to the multiparameter negative binomial and logarithmic series distributions of order $k$.

Proposition 3.3. Let $X_{i}(i \geq 1)$ be independent rvs distributed as $\mathrm{LS}_{k}\left(q_{1}, \ldots, q_{k}\right)$ independently of a $r v N$ which is distributed as $P_{1}(-r \log p)$, and set $S_{N}=X_{1}+\cdots+X_{N}$. Then $S_{N}$ is distributed as $\mathrm{NB}_{k}\left(r ; q_{1}, \ldots, q_{k}\right)$.

Proof. For $|t| \leq 1$, we have

$$
\begin{aligned}
g_{S_{N}}(t) & =g_{N}\left[g_{X_{1}}(t)\right]=\exp \left\{-r \log p\left[g_{X_{1}}(t)-1\right]\right\} \\
& =\exp \left\{-r \log p\left[-\alpha \log \left(1-\sum_{i=1}^{k} q_{i t^{i}}\right)-1\right]\right\} \\
& =\exp \left\{-r\left[\log \left(1-\sum_{i=1}^{k} q_{i t^{i}}\right)-\log p\right]\right\} \\
& =p^{r}\left(1-\sum_{i=1}^{k} q_{i} i^{i}\right)^{-r}
\end{aligned}
$$

which establishes the proposition, by means of Proposition 2.5(a).

The result just established may be written in the notation of Johnson and Kotz (1969) as

$$
\mathrm{NB}_{k}\left(r ; q_{1}, \ldots, q_{k}\right)=P_{1}(-r \log p) \vee \mathrm{LS}_{k}\left(q_{1}, \ldots, q_{k}\right) .
$$


We end this paper by giving one more genesis scheme for each one of the three multiparameter distributions of order $k$ treated presently. To this end, we recall the following definition of Hirano (1986).

DEFINITION 3.1. A rv $X$ is said to have the $k$-point distribution with parameters $v_{1}, \ldots, v_{k}$ to be denoted by $K\left(v_{1}, \ldots, v_{k}\right)$, if

$$
P(X=i)=v_{i}, \quad i=1, \ldots, k ; \quad 0<v_{i}<1, \quad \sum_{i=1}^{k} v_{i}=1 .
$$

The following proposition may be easily checked by means of Propositions 2.4(a)-2.6(a) and Definition 3.1.

PROPOSITION 3.4. Let $P_{k}\left(\lambda_{1}, \ldots, \lambda_{k}\right), \mathrm{NB}_{k}\left(r ; q_{1}, \ldots, q_{k}\right)$ and $\mathrm{LS}_{k}\left(q_{1}, \ldots\right.$, $q_{k}$ ) be given by Definitions $2.1-2.3$, respectively. Set $\lambda=\lambda_{1}+\cdots+\lambda_{k}$ and $q=$ $q_{1}+\cdots+q_{k}$. Then
(a) $P_{k}\left(\lambda_{1}, \ldots, \lambda_{k}\right)=P_{1}(\lambda) \vee \mathrm{K}\left(\lambda_{1} / \lambda, \ldots, \lambda_{k} / \lambda\right)$;
(b) $\mathrm{NB}_{k}\left(r ; q_{1}, \ldots, q_{k}\right)=\mathrm{NB}_{1}(r ; q) \vee \mathrm{K}\left(q_{1} / q, \ldots, q_{k} / q\right)$;
(c) $\operatorname{LS}_{k}\left(q_{1}, \ldots, q_{k}\right)=\operatorname{LS}_{1}(q) \vee \mathrm{K}\left(q_{1} / q, \ldots, q_{k} / q\right)$.

\section{Acknowledgement}

The author wishes to thank an anonymous referee for his helpful comments which led to Remark 2.1 and improvements in the presentation of the paper.

\section{REFERENCES}

Adelson, R. M. (1966). Compound Poisson distributions, Operational Research Quarterly, 17, 73-75.

Aki, S. (1985). Discrete distributions of order $k$ on a binary sequence, Ann. Inst. Statist. Math., 37, 205-224.

Aki, S., Kuboki, H. and Hirano, K. (1984). On discrete distributions of order $k$, Ann. Inst. Statist. Math., 36, 431-440.

Charalambides, Ch. A. (1986). On discrete distributions of order $k$, Ann. Inst. Statist. Math., 38, 557-568.

Hirano, K. (1986). Some properties of the distributions of order $k$, Fibonacci Numbers and Their Applications: Proceedings of the First International Conference on Fibonacci Numbers and Their Applications (Patras 1984), (eds. A. N. Philippou, G. E. Bergum and A. F. Horadam), 45-53, Reidel, Dordrecht.

Johnson, N. L. and Kotz, S. (1969). Discrete distributions, Houghton-Mifflin, New York.

Philippou, A. N. (1983). Poisson and compound Poisson distributions of order $k$ and some of their properties, Zap. Nauchn. Sem. Leningrad. Otdel. Mat. Inst. Steklov., 130, 175-180 (in Russian, English summary).

Philippou, A. N. (1984). The negative binomial distribution of order $k$ and some of its properties, Biometrical $J$., 26, 789-794. 
Philippou, A. N., Georghiou, C. and Philippou, G. N. (1983). A generalized goemetric distribution and some of its properties, Statist. Probab. Lett., 1, 171-175.

Philippou, A. N. and Makri, F. S. (1986). Successes, runs and longest runs, Statist. Probab. Lett., 4, 211-215.

Philippou, A. N. and Muwafi, A. A. (1982). Waiting for the $k$ th consecutive success and the Fibonacci sequence of order $k$, Fibonacci Quart., 20, 28-32.

Xekalaki, E., Panaretos, J. and Philippou, A. N. (1987). On some mixtures of distributions of order k, Fibonacci Quart., 25, 151-160. 